\title{
The Simulation of a PEMFC with an Interdigitated Flow Field Design
}

\author{
S.M. Guo \\ Dept. Mechanical Engineering, \\ Louisiana State University, \\ Baton Rouge, LA 70803, USA \\ $+12255787619$ \\ sguo2@lsu.edu
}

\begin{abstract}
This paper presents the simulations for a two-dimensional PEMFC with an interdigitated flow channel design using FEMLAB. The multi-species flow of $\mathrm{O}_{2}, \mathrm{H}_{2}, \mathrm{H}_{2} \mathrm{O}$ and inert $\mathrm{N}_{2}$ is examined over the entire fuel cell working range. The transportations of these gases in the porous anode/cathode are predicted using the Maxwell-Stefan transport equations and the Fick's law; the flow field is predicted using the Darcy's law; and the electrical field is simulated using a conductive media model. The standard current-voltage performance curve, the species concentration of $\mathrm{O}_{2}, \mathrm{H}_{2}, \mathrm{H}_{2} \mathrm{O}$ and inert $\mathrm{N}_{2}$, mass fluxes, electrical current and potential distributions have been obtained.
\end{abstract}

\section{Introduction}

Fuel cells are electrochemical devices, which utilize fuel and oxidant to produce electricity and heat [1]. Because the fuel is converted chemically to electricity, fuel cells may operate at higher efficiencies than conventional internal combustion engines. Fuel cells are classified by the electrolytes. The common electrolyte of a Proton Exchange Membrane Fuel Cell (PEMFC) is a thin layer of proton permeable polymer membrane. In a PEMFC system, the coupled physical and electrochemical processes take place. Transport resistances of the gaseous species in the feeding channels and the porous electrodes lead to the so-called concentration over-potential; the transportation of $\mathrm{H}^{+}$in the electrolyte layer forms a large portion of ohmic loss; the activation energy barriers for the electrochemical reactions are related to the charge-transfer processes at the electrode-electrolyte interfaces. The theoretical potential of a hydrogen/oxygen cell, operating under standard conditions of $1 \mathrm{bar}$ and $25^{\circ} \mathrm{C}$, is about 1.2 volt. However, due to losses, the voltage of a single cell is much less than its theoretical value.

The PEMFC flow-field, including the feeding channels and the porous electrodes, has a significant influence to the cell performance. The performance of a fuel cell is often described by its current-voltage (I-E) relationship. Fuel, such as hydrogen, and small amount of water vapor are supplied to the PEMFC anode side. Due to the electrochemical reactions, steam forms at the cathode. For a typical planar PEMFC design, feeding channels are provided by the bipolar plates with a typical serpentine 
or parallel channel structure. Due to the long channels, serpentine flow-fields have large pressure losses between the inlet and the outlet. Although straight parallel design exhibits lower pressure differences, inhomogeneous reactant gas distribution can easily occur. These flow channels must distribute fuel and air over the reaction sites and remove the products. Recently, a number of novel flow channels have been proposed for Proton Exchange Membrane Fuel Cells, in order to address the PEMFC mass transport and water management problems. The most promising design is the interdigitated flow field design $[2,3,4]$. Figure 1 shows the schematic drawing of this design. The flow channels are dead-ended, forcing gas to flow through the porous diffusion layer. Comparing to the flow in a conventional straight parallel flow field design, the use of the interdigitated fuel/air distributors imposes a pressure gradient between the inlet and the outlet channels, forcing the convective flow of the fuel/oxidant through the porous electrodes. Thus the interdigitated design in effect converts the transport of gases to/from the Triple Phase Boundary (TPB) active sites, along the interface between the electrodes and electrolyte, from a diffusion dominated mechanism to a forced convection dominated mechanism. The convective flow through the porous electrode reduces the gas diffusion distance to and from the reaction sites. By having fuel/oxidant flow over the shoulders of the gas distributor, the electrode active area over the shoulder is used more effectively. The shear force of the gas stream helps removing the water condensate, which is entrapped in the electrode layer, thereby reducing the flooding problem. This design has been proven to be very effective by some experimental studies. Wang et al. [3] presented an experimental study of PEM fuel cells with interdigitated flow fields under different operation parameters. Nguyen [4] presented a comparative experimental study of fuel cells with interdigitated flow fields and parallel straight channel flow fields. They reported that the interdigitated flow design could extend the PEMFC operable regime to higher current densities and consequently, a 50-100\% increase in the fuel-cell performance could be obtained as a result of the use of interdigitated fuel/air distributors. To study a fuel cell performance mathematically, especially for a design with thick porous electrodes and a strong convective flow, a proper mass transport model must be applied. At high current density, the losses are dominated by the limitation of transport the fuel/oxidant to the reaction sites, the so-called concentration or mass transport over-potential. Mass transport in the porous electrodes depends on the structure of the porous electrodes, such as the porosity, tortuosity and mean pore size. Washak et al. [5] and Suwanwarangku et al. [6] conducted comparative studies using the Fick's law and the Dusty Gas Model for a Solid Oxide Fuel Cell. They found that the current density, the reactant concentration and the pore size were the three key parameters for choosing a proper porous media simulation model. The Dusty Gas Model works better for the $\mathrm{H}_{2}-\mathrm{H}_{2} \mathrm{O}$ and $\mathrm{CO}-\mathrm{CO}_{2}$ systems, especially under high operating current densities, low reactant concentrations and small pore sizes.

To perform a parametrical optimization for the flow field design, multiphysics based numerical simulation offers many advantages comparing to the experimental approach.

The aim of this paper is to study the effect of using different mass transport models on the fuel cell performance simulation. Two models are examined in this paper. The first one is the Fick's model, and the second model applies the Stefan-Maxwell 
diffusion equations to the mixture. The performance of a PEMFC with an interdigitated flow channel design was simulated using FEMLAB. The simulation includes the multi species transportation in the porous electrodes and the coupled electrical current and potential distributions.

\section{The Simulation Model}

FEMLAB is a commercial Partial Differential Equation (PDE) solver, which can solve coupled multi-physical problems. Partial differential equations are the governing equations for most physical phenomena and provide the foundation for modeling a wide range of scientific and engineering problems. There are three ways of describing PDEs in FEMLAB, coefficient form, general form and the weak form. The coefficient form is suitable for linear or nearly linear models and the rest two are suitable for nonlinear models. FEMLAB runs finite element analysis to solve the PDEs, together with adaptive meshing and error controls.

The simulation domain used in this paper is a 2-dimensional cross section of an interdigitated PEMFC flow field, shown in Figure 1. The oxidation of hydrogen and the reduction of oxygen take place at the anode and cathode side reaction boundaries respectively. Electrons are transported to an outer circuit at the anode and received at the cathode because only proton ions can pass through the electrolyte membrane.

$$
\begin{aligned}
& \mathrm{H}_{2} \rightarrow 2 \mathrm{H}^{+}+2 e^{-} \text {at anode side reaction boundary } \\
& \mathrm{O}_{2}+4 \mathrm{H}^{+}+4 e^{-} \rightarrow 2 \mathrm{H}_{2} \mathrm{O} \text { at the cathode side reaction boundary }
\end{aligned}
$$

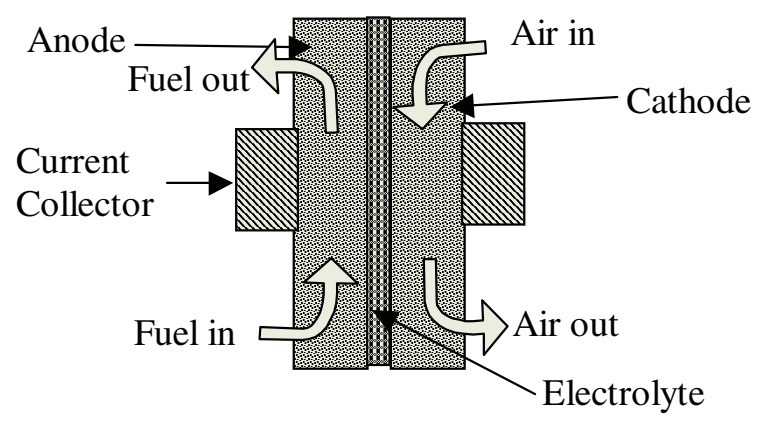

Fig. 1. Interdigitated Flow Field Design

The thickness of the anode and cathode is set to be $0.25 \mathrm{~mm}$ and the height is set to be $2 \mathrm{~mm}$; the electrolyte layer has a thickness of $0.1 \mathrm{~mm}$. Along the height direction, current collectors of $1 \mathrm{~mm}$ in length are in contact with the outer surface of the anode and cathode in a symmetrical manner, leaving the inlet and outlet ports a dimension of $0.5 \mathrm{~mm}$. Hydrogen and an inert gas mixture is fed into the anode side inlet port while oxygen, inert nitrogen and water are fed into the cathode side. The gas mixtures are treated as incompressible due to the low flow velocities. In the simulation, the 
hydrogen is oxidized along the electrode/electrolyte interface, which has a zero thickness. The electrical potential of the PEMFC is specified as an input parameter over the entire working range.

A set of PEMFC governing equations is specified in FEMLAB. Equation 1 is the steady state continuity equation.

$$
\nabla \bullet(C V)=0
$$

Where $C$ is the total gas mixture molar concentration; $V$ is the velocity; $C V$ is the total molar flux. For continues gas phase flow in the porous electrodes, Darcy's law, Equation 2, is used.

$$
V=-\frac{k_{p}}{\mu} \nabla P
$$

Where, $k_{p}$ is the permeability of the medium, $\mu$ is the dynamic viscosity of the gas mixture, $\nabla P$ gives the pressure gradient.

For the multi species mass transfer in the electrodes, both Fick's law and the Maxwell-Stefan diffusion and convection mass transfer models were tested. Using the Fick's law, the diffusion flux in the porous electrodes is calculated using equation 3.

$$
N^{d}=-D^{e} \nabla C
$$

$D^{e}$ is the effective diffusivity of the gas, $C$ is the concentration. By assuming equal counter-current molar fluxes, according to Chan et al. [7], the composition independent $D^{e}$ can be found in Eq. 4

$$
D_{1}^{e}=\left(\frac{1}{D_{12}^{e}}+\frac{1}{D_{1 k}^{e}}\right)^{-1}
$$

$D_{12}^{e}$ is the effective binary bulk diffusion coefficient. $D_{1 k}^{e}$ is the effective Knudsen diffusion coefficient, which depends on temperature and structure of the porous material. For a convective flow in a porous medium, combining the diffusive and convective fluxes, the flux equation can be written as equation 5 .

$$
N=-\left(\frac{1}{D_{12}^{e}}+\frac{1}{D_{1 k}^{e}}\right)^{-1} \nabla C-\frac{X_{1}}{R T} \frac{k_{p} P}{\mu} \nabla P
$$

For the simulation using the Maxwell-Stefan diffusion and convection model. The Maxwell-Stefan multi-component diffusion is given in equation 6.

$$
\frac{\partial}{\partial t} \rho \omega_{i}+\nabla \bullet\left[-\rho \omega_{i} \sum_{j=1}^{N} D_{i j}\left\{\begin{array}{l}
\frac{M}{M_{j}}\left(\nabla \omega_{j}+\omega_{j} \frac{\nabla M}{M}\right) \\
+\left(x_{j}-\omega_{j}\right) \frac{\nabla P}{p}
\end{array}\right\}+\omega_{i} \rho u+D_{i}^{T} \frac{\nabla T}{T}\right]=R_{i}
$$


where $D_{i j}$ is the diffusion coefficient $\left(\mathrm{m}^{2} / \mathrm{s}\right), P$ the pressure $(\mathrm{Pa}), T$ is the temperature $(\mathrm{K}), u$ the velocity vector $(\mathrm{m} / \mathrm{s}), x$ and $\omega$ are mole and mass fractions. The density, $\rho$ $\left(\mathrm{kg} / \mathrm{m}^{3}\right)$, is calculated based on the mole fractions and mole masses of gas species.

At inlet, pressure and the feeding gas mass fractions are specified. At the outlets, a convective flux boundary condition is applied. The local current density is a function of the local species concentration, physical structure of the electrodes and the specified cell output electrical potential. For steady state calculations, due to the conservation of current, the anode side current density is the same as the current density at the cathode. At the anode and cathode reaction boundary, the species mass transfer is related to the local current density according to: $-n \bullet n_{H_{2}}=-\frac{l_{a}}{2 F}$ at anode reaction boundary and $-n \bullet n_{O_{2}}=\frac{i_{C}}{4 F}$ at the cathode reaction boundary.

The potential difference between the cathode and anode current collectors represents the cell voltage. In the simulation, the potential at the anode current collector was arbitrarily chosen to be zero, while the cell voltage at the cathode current collector is set as a fixed boundary condition. The potential distributions in the anode, cathode and the electrolyte are modeled as conductive media using equation7.

$$
\nabla \bullet(-k \nabla E)=0
$$

where $k$ is the effective conductivity $(\mathrm{S} / \mathrm{m})$ and $E$ is the potentials in the calculation domain. The rest of the boundaries were set as the electrical insulators or as a symmetrical boundary. Normal to the reaction boundary, $n \bullet(-k \nabla E)$ gives the current density.

\section{Results and Discussions}

The $I-E$ performance curve of a PEMFC has been obtained using two different mass transfer models, Fick's model and the Maxwell-Stefan model. Figure 2 shows a typical fuel and oxidant distribution inside a PEMFC with an interdigitated flow field design (see Fig. 1 for feeding directions). A $60 \% \mathrm{H}_{2}$ is fed to the anode side (left half) and a $21 \% \mathrm{O}_{2}$ is fed to the cathode (right half) inlet port.

As expected, the Fick's model results show discrepancies to the Maxwell-Stefan model results in the high current density region, see Fig. 3. Start at about $3000 \mathrm{~A} / \mathrm{m}^{2}$, Fick's law started to over-predict the current density at fixed cell output electrical potentials. At the high current end, the difference between these two models is about $15 \%$. This is mainly caused by the over prediction of the oxygen concentration at the cathode side reaction boundary. The oxygen concentration at the inlet is $21 \%$. Due to the electrical chemical reactions along the cathode/electrolyte boundary, the oxygen concentration at the reaction sites is far less than the inlet value, see Fig. 2. Figure 4 shows the predicted oxygen concentration average along the cathode reaction boundary under different current densities using those two models. The difference between the two models is obvious at the high current region, where the Fick's model predicts a higher $\mathrm{O}_{2}$ concentration. Figure 5 shows the production of water due to the 
electrochemical reactions. The curve shows a strong linear relationship between the water formation and the current density.

The definition of anode side $\mathrm{H}_{2}$ concentration is based on the hydrogen partial pressure. Because of the electrochemical reactions, hydrogen depletes along the flow direction. The mass transport process affects the local concentration of fuel. Hydrogen concentration reduces almost linearly with the increase of current density. At about $6500 \mathrm{~A} / \mathrm{m}^{2}$, the right end of the curve, the hydrogen mass transfer in the porous anode reaches its limiting current density.

The commercial FEMLAB software has many built-in multi physics models. However, the advantage of a general software package, which claims to solve "many" physical problems, is also likely to be its weakness. The build-in models are generally

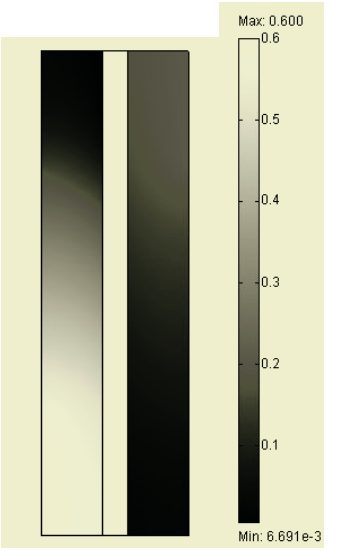

Fig. 2. A typical $\mathrm{H}_{2}$ (left) and $\mathrm{O}_{2}$ (right) concentration in the PEMFC

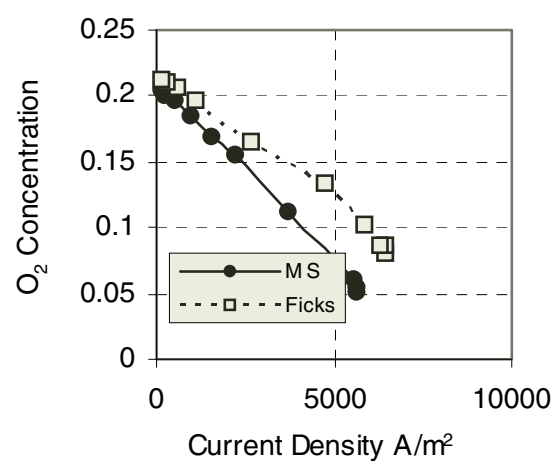

Fig. 4. PEMFC cathode reaction boundary $\mathrm{O}_{2}$ concentration predicted using the Fick's model and the Maxwell-Stefan model

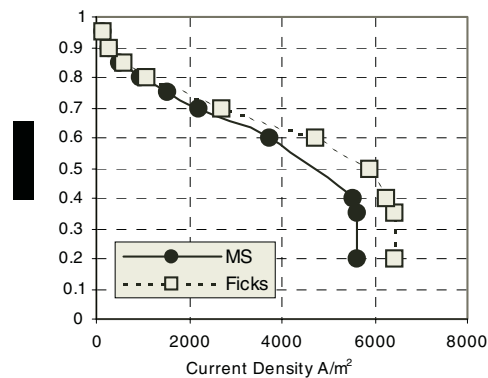

Fig. 3. PEMFC $I-E$ curves predicted using the Fick's model and the Maxwell-Stefan model

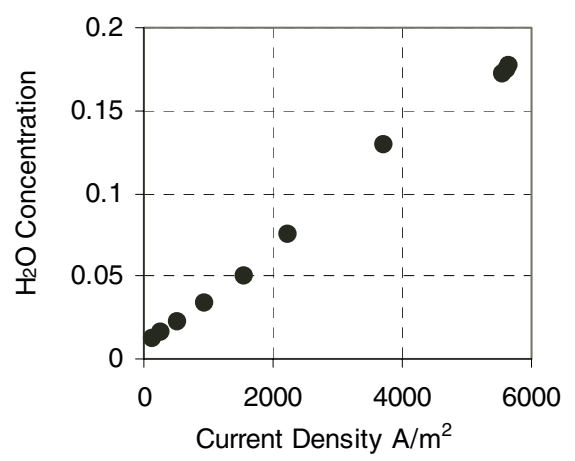

Fig. 5. PEMFC cathode reaction boundary $\mathrm{H}_{2} \mathrm{O}$ concentration as a function of cell current density 


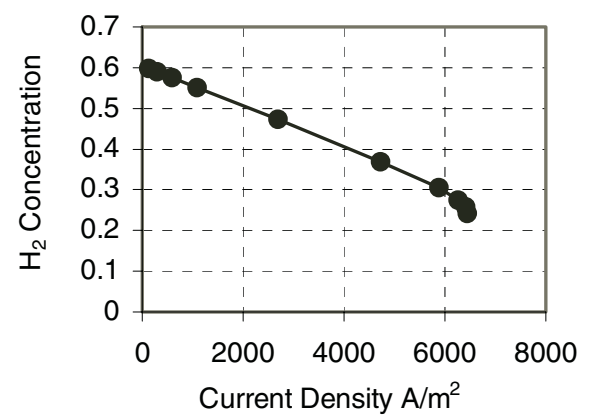

Fig. 6. PEMFC anode reaction boundary $\mathrm{H}_{2}$ concentration

to be the basic physical and mathematical models for a particular physical problem. For example, because only Darcy's law was used in the simulation to predict the fluid flow in the porous electrodes, the accuracy near the reaction boundaries is not expected to match the accuracy of a well-tuned CFD case, which uses Navier-Stokes equations with proper turbulent models. To solve a real multi-physical problem, a balanced approach must be carefully taken. The number of physical phenomena, to be modelled in software, must be considered in conjunction with the expected overall accuracy and the available computing power.

\section{Conclusions}

In the past two decades, due to the energy shortage and the environmental concerns, great efforts have been put into fuel cell research. To improve the fuel cell performance, multiphysics analysis could be employed. Using proper fuel cell models, fuel cell simulations could provide detailed understanding and solutions on how to reduce the losses and how to improve the operating efficiency. Comparing with an experimental based approach, computational simulations are low cost and could quickly provide the physical understandings to a particular fuel cell design. The PEMFC flow-field, including feeding channels and porous electrodes, has significant influence to the cell performance. The local concentration of fuel and oxidant is affected by the mass transport processes, which include the mixing of multiple gases in the porous anode/cathode, the reactions of fuel and oxygen and the transportation of fuel and electrochemical products near the reaction sites. This paper presents the numerical simulations of gas transport in the porous electrodes of a PEMFC with an interdigitated flow channel design using FEMLAB. The simulation is a coupled fluid dynamics and electrical potential field problem with multi species mass transfer and chemical reactions. This complicated PEMFC problem has been solved using FEMLAB over the entire working range. Two porous media mass transport models, the Fick's Model and the Maxwell-Stefan model were used in the simulations for multi-species of $\mathrm{O}_{2}, \mathrm{H}_{2}, \mathrm{H}_{2} \mathrm{O}$ and inert $\mathrm{N}_{2}$. The standard current-voltage performance curve and the detailed species concentration, mass fluxes, electrical current and 
potential distributions have been obtained. The simple but less accurate Fick's model is validated against the Maxwell-Stefan model. Fick's model was found to overpredict the oxygen concentration along the cathode side reaction boundary and thus over-predict the cell performance in the high current density region.

\section{References}

1. Larminie, J., Dicks, A., Fuel cell systems explained, Wiley, ISBN: 047084857x, (2003)

2. Jung, S. Y., Nguyen, T.V., Multicomponent Transport in Porous Electrodes of Proton Exchange Membrane Fuel Cells Using the Interdigitated Gas Distributors, Journal of The Electrochemical Society, v 146, n1, 1999, p 38-45

3. Wang, L., Liu, H., Performance studies of PEM fuel cells with interdigitated flow fields, Journal of Power Sources, 134, p185-196, (2004)

4. Nguyen, T.V., Gas distributor design for proton-exchange-membrane fuel cells, J. Electrochem. Soc. 143, n 5, p L103-L105, (1996)

5. Washak, H., Guo, S.M., Turan, A., 2005, Gas Transport in Porous Electrodes of Solid Oxide Fuel Cells, the Ninth International Symposium on Solid Oxide Fuel Cells (SOFC IX), The 207th Meeting of The Electrochemical Society, Québec City Convention Centre, May 15-20, 2005

6. Suwanwarangkul, R., Croiset, E., Fowler, M.W., Douglas, P.L., Entchev, E., Douglas, M.A., Performance comparison of Fick's, dusty-gas and Stefan-Maxwell models to predict the concentration overpotential of a SOFC anode, Journal of Power Sources 122 9-18, (2003)

7. Chan, S.H., Khor, K.A., Xia, Z.T., A complete polarization model of a solid oxide fuel cell and its sensitivity to the change of cell component thickness, Journal of Power Sources, 93, 130-140, (2001) 